\title{
BMJ Open Defining timeliness in care for patients with lung cancer: protocol for a scoping review
}

\author{
Adnan Ansar (D) , ${ }^{1}$ Virginia Lewis, ${ }^{2}$ Christine Faye McDonald, ${ }^{3}$ Chaojie Liu (i) , ${ }^{4}$ \\ Aziz Rahman ${ }^{5,6}$
}

To cite: Ansar A, Lewis V, McDonald CF, et al. Defining timeliness in care for patients with lung cancer: protocol for a scoping review. BMJ Open 2020;10:e039660. doi:10.1136/ bmjopen-2020-039660

- Prepublication history and supplemental material for this paper is available online. To view these files, please visit the journal online (http://dx.doi. org/10.1136/bmjopen-2020039660).

Received 22 April 2020 Revised 27 July 2020

Accepted 24 September 2020

\section{Check for updates}

(c) Author(s) (or their employer(s)) 2020. Re-use permitted under CC BY-NC. No commercial re-use. See rights and permissions. Published by BMJ.

${ }^{1}$ College of Science, Health and Engineering, La Trobe University, Melbourne, Victoria, Australia

${ }^{2}$ Australian Institute for Primary

Care and Aging, La Trobe

University, Bundoora, Victoria,

Australia

${ }^{3}$ Respiratory and Sleep

Medicine, Austin Health, Heidelberg, Victoria, Australia

${ }^{4}$ School of Psychology and

Public Health, La Trobe

University, Bundoora, Victoria,

Australia

${ }^{5}$ School of Health, Federation University Australia, Berwick,

Victoria, Australia

${ }^{6}$ School of Nursing and

Midwifery, La Trobe University,

Melbourne, Victoria, Australia

Correspondence to

Dr Adnan Ansar;

ansar.a@students.latrobe.

edu.au

\section{ABSTRACT}

Introduction Cancer is the second leading cause of death worldwide, and lung cancer is the single leading cause of cancer mortality worldwide. Early diagnosis of lung cancer is the key to better prognosis and longer survival. While there are substantial literature reporting delays in cancer diagnosis, there is a lack of consensus in the definitions and terms used to describe 'delay' in the treatment pathway. The aim of this scoping review is to identify and critically synthesise the operational definitions and terminologies used to describe the timely initiation of care and consequent treatments over the care pathway for patients with lung cancer. This scoping review will also compare how timeliness was operationalised in Western and Asian countries.

Methods and analysis The scoping review will use the methodology described by Arksey and O'Malley and endorsed by the Joanna Briggs Institute. MEDLINE, EMBASE, CINAHL and PsycINFO electronic databases will be searched. Grey literature sources and the reference lists of key studies will be used to identify additional relevant studies. The scoping review will include all studies, irrespective of study methodology and quality. Two reviewers will independently screen all titles and abstracts to identify eligible studies for inclusion. The full texts of identified studies will be further examined and charted using a data extraction form. A narrative synthesis will be performed to assess and categorise available definitions of timeliness.

Ethics and dissemination Ethical approval is not needed as this scoping review will be reviewing already published articles. The results produced from this review will be submitted to a scientific peer-reviewed journal for publication and will be presented at scientific meetings.

\section{BACKGROUND}

Lung cancer is the leading cause of cancer mortality worldwide, with an estimated 2.09 million deaths in 2018. ${ }^{1}$ It is considered to be the most aggressive human cancer, with a 5-year survival rate of 10\%-15\% overall and only $2 \%$ in stage IV. ${ }^{2}$ Moreover, survival rates may vary from country to country due to insufficient population coverage of healthcare despite recent advancements in oncology. ${ }^{3}$

Early diagnosis is the key to ensuring timely treatment and a better prognosis. ${ }^{4}$ Despite

\section{Strengths and limitations of this study}

The scoping review will document the heterogeneity in the definitions of timely initiation of care and consequent treatments over the care pathway for patients with lung cancer across different healthcare settings.

- This scoping review will compare how timeliness is operationalised in research in Western and Asian countries.

- Only studies published in English will be included in the review, which may miss potential literature in other languages.

the existence of guidelines that include recommended timepoints to diagnosis and treatment along a pathway to care for lung cancer, there is inconsistency and ambiguity in how the standard timeline to diagnosis (from first symptom and first interaction with a healthcare professional, first suggestive investigation, diagnosis) and treatment should be defined. ${ }^{5}$

Importantly, the timeframe and pathway to care recommended in standard guidelines which underpin practices in the USA, ${ }^{67}$ Australia, ${ }^{8}$ the $\mathrm{UK}^{9}$ and other European countries ${ }^{10}$ have been developed in the context of high functioning health systems with established referral mechanisms. It is unlikely that these guidelines can be fully implemented for optimal effectiveness in developing countries as the health systems are often weak, with limited resources and health coverage as well as underdeveloped referral options or processes. ${ }^{11}$ Thus, developing countries are likely to require different sets of definitions for timely care and guidelines that align with the characteristics of the respective health systems. ${ }^{12}$

Despite the Aarhus statement and checklist to guide design and reporting of research into early diagnosis, ${ }^{13}$ there is a lack of consistency in the definitions and terminologies used to 
describe timeliness of seeking and receiving care for lung cancer in research. The lack of widespread adoption of proposed definitions may reflect experiences in different health systems in which research has been conducted. A number of different care timepoints and intervals between them have been identified in different timeliness of lung cancer care-related studies, such as initial presentation, decision to seek care, first contact with a healthcare provider, referral to a specialist, first investigation, first imaging suggestive of cancer, diagnosis, first treatment, day of surgical intervention, first day of radiation, first day of chemotherapy and day started palliative care. ${ }^{14-16}$ Studies have used different metrics and measurement units to report gaps or intervals between timepoints in mean, median or percentile. ${ }^{14-16}$ As a result, timeliness-related publications often leave the readers to make assumptions about the proper meaning of the terms and findings.

A lack of consensus on which intervals between timepoints to measure and how to report them makes comparisons between studies difficult. Moreover, research on operational definitions or terminologies in this area is also scarce. Given the inconsistency in the terminology and variations in intervals between timepoints, the heterogeneity of study designs in this area, along with the differences between health systems in different countries, a scoping review will be undertaken to assess and categorise all available definitions and terminologies. The review will include clinically relevant timepoints of seeking or receiving care used in different studies conducted in various countries. A scoping review approach is considered to be the best way to identify and include potentially informative articles with maximum inclusivity.

The aim of this scoping review is to identify and critically synthesise the operational definitions and terminologies used to describe the timeliness of seeking and receiving care for lung cancer and the intervals between timepoints used to describe the care-seeking pathway in different studies conducted in different healthcare settings. The review will also explore differences in how timely care seeking and receiving is defined in Western and Asian countries.

\section{METHODS}

The methodological framework for conducting this scoping review will be based on Arksey and O'Malley's methodological framework, ${ }^{12}$ which was further clarified by Levac et $a l^{17}$ and the Joanna Briggs Institute. ${ }^{18}$ As recommended by Arksey and O'Malley, the review process will consist of the following:

Stage 1. Identifying the research question.

Stage 2. Identifying relevant studies.

Stage 3. Study selection.

Stage 4. Charting the data.

Stage 5. Collating, summarising and reporting the results.

Stage 6. Consultation with key stakeholders.
Stage 6 is optional according to Arksey and O'Malley and 'provides opportunities for consumer and stakeholder involvement to suggest additional references and provide insights beyond those in the literature'. Stage 6 will not be part of the current scoping review, as the focus of the review is on the use of terms in the literature rather than seeking broader insights into timeframes and delay. This scoping review will capture and categorise available definitions and terminologies of timeliness without an intention of achieving consensus.

The methods to be used in this scoping review protocol will be rigorous and transparent with an attempt to document the processes in sufficient detail to ensure replicability. The University of York Centre for Reviews and Dissemination guidance for undertaking reviews in healthcare ${ }^{19}$ and the PRISMA-ScR (Preferred Reporting Items for Systematic Reviews and Meta-Analyses for Scoping Review) checklist ${ }^{20}$ will be followed to ensure the comprehensiveness of the protocol as well as standardisation and reporting.

\section{STAGE 1: IDENTIFYING THE RESEARCH QUESTION}

The research questions emerged from a literature review undertaken for a project assessing the timeliness of seeking care of patients with lung cancer in a developing country. It was evident that different studies adapted operational definitions from similar studies without clearly defining the parameters for timeliness and the stages or intervals in the treatment pathway. Hence, the aim of this scoping review is to assess and categorise the definitions and terminologies used to describe timeliness of seeking and receiving care in patients with lung cancer in published articles and grey literature. To address this aim, the following research questions will be asked:

1. What are the existing definitions and terminologies used to describe timeliness of seeking and receiving care for lung cancer in the literature?

2. What are the timepoints and intervals identified in the care pathway for lung cancer under different health systems in the literature?

3. Are there differences in the definitions and terminologies used in the literature from Western and Asian countries?

\section{STAGE 2: IDENTIFYING RELEVANT PUBLICATION}

This scoping review will attempt to address the research questions comprehensively.

\section{Inclusion criteria}

The scoping review will include all studies, irrespective of study methodology and quality, ${ }^{21}$ to gain a better understanding of how researchers have defined and measured timeliness of seeking and receiving care for lung cancer in various countries and healthcare settings. Both peerreviewed publications and grey literature will be included. 


\section{Population}

The population of this scoping review will be patients with lung cancer, diagnosed by clinicians irrespective of histological type and disease stage.

\section{Concept}

Terminologies and definitions used to describe timeliness of seeking care, timepoints in seeking care, intervals between timepoints in the disease care pathway, including patient's first symptom appearance, decision to seek care, first contact with a health professional to diagnosis and diagnosis to initiation of treatment of patients with lung cancer.

\section{Context}

Studies, reports, guidelines from any country and reporting from the perspectives of patients and healthcare providers.

\section{Publication type and status}

In order to avoid publication bias, this scoping review will include all relevant studies, regardless of publication type (editorials, book chapters, reports, original articles, review articles, theses, conference abstracts) and status (published or accepted for publication and grey literature). Previous reviews on similar topics will be included and the reference list will be screened for relevant articles.

\section{Language}

Considering the timeframe, resources and facilities for translation, only studies published in English will be included. From the initial search in MEDLINE, 5\% of publications were found to be in another language other than English.

\section{Timeframe}

Articles published in the last 20 years will be included. This timeframe has been chosen because there have been substantial advancements in lung cancer management in the last two decades, such as use of low-dose CT for screening, ${ }^{22}$ updating the staging criteria, immunotherapy, targeted therapy and their combination with chemotherapy, radiotherapy $^{23}$ and increase in surgical resection. ${ }^{24}$ The influence of evidence-based medicine on recent updates of treatment guidelines also contributed to the decision to focus on the past 20 years. The intended start date is April 2020 and the review should be completed by October 2020.

\section{Exclusion criteria}

Studies will be excluded if timeliness of care or timepoints and intervals in the care pathway are not reported, reported delays are not specific for lung cancer, or the primary focus of the article was not timeliness of care or the different timepoints of seeking care. Studies reporting clinical trials of drugs, non-human subjects and studies published only as abstracts will also be excluded.

\section{Databases}

MEDLINE, EMBASE, PsycINFO and CINAHL will be searched for published articles. The Google search engine will be used to search for editorials, reports and grey literature. Particular attention will be paid to existing standard guidelines for lung cancer care timeframes recommended in the countries that lead in this area, such as the USA, Australia, the UK and European countries. Grey literature databases will be searched (eg, Grey Literature Report) to identify studies, reports and conference abstracts of relevance to this review.

MEDLINE, EMBASE and PsycINFO will be searched using the Ovid interface, and CINAHL will be searched using EBSCOhost interface.

\section{Search strategy for electronic databases}

The search strategy for the selected database will follow the three-step process recommended by the Joanna Briggs Institute. In the first step, an initial preliminary search of two online databases (MEDLINE and EMBASE) relevant to the topic will be done. The text contained in the titles and abstracts of the papers from the search, and the key words used to describe the articles will be analysed. In the second step, all the identified keywords and index terms will be used to formulate the final search strategies specific to the selected databases (table 1 ). In the third step, additional studies will be searched from the reference list of identified reports and articles included (table 2).

Table 1 Identified keywords and index terms to construct search strategy for the scoping review

\begin{tabular}{lll}
\hline Lung cancer-related terms & Care-seeking-related terms & Timeliness-related terms \\
\hline Lung neoplasms/Carcinoma, & General practitioner/General practice/ & Diagnostic timelines/Delay/Early detection of \\
Non-Small-Cell Lung/ & Family practice/Family physician/Primary & cancer/Delayed detection of cancer/Primary delay/ \\
Carcinoma, Small Cell Lung/ & healthcare/Secondary healthcare/Tertiary & Secondary delay/Tertiary delay/Health system delay/ \\
Respiratory Tract Neoplasms/ & healthcare/Public hospitals/Private & Timeliness/Time factor/Interval/ Patient interval/ \\
Lung adenocarcinoma/Lung & hospitals/Special hospitals Cancer & Patient delay/Clinician delay/Physician delay/Referral \\
cancer symptom & palliative care/ Pulmonologist/Oncologist/ & delay/Diagnosis delay/ Diagnostic delay/Diagnostic \\
& Thoracic surgery/Thoracotomy Lung & evaluation/Delayed diagnosis/Time to treatment/ \\
& lobectomy/Pneumonectomy/Surgical & Treatment initiation/Treatment delay/Wait time/ \\
& resection/Referral/Referral and & Wait time intervals/Waiting list/Appointment and \\
& consultation/Delivery of health care/ & schedules/Health service accessibility/Help seeking \\
& Patient admission & intervals/Prognostic implication/Lung cancer survival \\
\hline
\end{tabular}


Table 2 Search strategy for different database

\begin{tabular}{|c|c|}
\hline Database & Search strategy \\
\hline Medline & $\begin{array}{l}\text { exp Lung Neoplasms/OR exp Carcinoma, Non-Small-Cell Lung/OR exp Carcinoma, Small Cell/OR adenocarcinoma/OR exp } \\
\text { adenocarcinoma, bronchiolo-alveolar/OR exp pulmonary adenomatosis, ovine/AND General Practitioners/OR Family Practice/O } \\
\text { General Practice/OR Primary Health Care/OR Secondary healthcare.mp. OR Patient Admission/OR exp Tertiary Healthcare/OR } \\
\text { Hospitals, Public/OR Hospitals, Private/OR Hospitals, Special/OR Palliative Care/OR exp Pulmonologists/OR exp Oncologists/ } \\
\text { OR exp surgical oncology/OR exp thoracic surgery/OR 'Referral and Consultation'/AND Diagnostic timelines.mp. OR Delay.mp. } \\
\text { OR exp 'Early Detection of Cancer'/OR Primary delay.mp. OR Secondary delay.mp. OR Tertiary delay.mp. OR Health system } \\
\text { delay.mp. OR Timeliness.mp. OR Interval.mp. OR Patient interval.mp. OR Patient delay.mp. OR Clinician delay.mp. OR Physicia } \\
\text { delay.mp. OR * 'Referral and Consultation'/OR Referral delay.mp. OR exp *Delayed Diagnosis/OR Diagnosis delay.mp. OR } \\
\text { Diagnostic evaluation.mp. OR exp *Time-to-Treatment/OR Treatment initiation.mp. OR Treatment initiation.mp. OR Treatment } \\
\text { delay.mp OR exp *Waiting Lists/OR Wait time.mp. OR exp * 'Appointments and Schedules'/OR Wait time intervals.mp. OR } \\
\text { Help seeking intervals.mp. OR *Prognosis/OR Lung cancer Survival.mp. OR Prognostic implication.mp. AND limit } 43 \text { to (English } \\
\text { language and humans and last } 20 \text { years) }\end{array}$ \\
\hline Embase & $\begin{array}{l}\text { exp lung tumor/OR exp non-small cell lung cancer/OR exp small cell lung cancer/OR exp lung adenocarcinoma/AND General } \\
\text { Practitioners.mp. or exp general practitioner/OR exp primary health care/OR exp secondary health care/OR exp tertiary health } \\
\text { care/OR exp public hospital/OR exp private hospital/OR exp cancer center/OR exp palliative therapy/OR exp pulmonologist/ } \\
\text { OR exp thoracotomy/OR exp lung lobectomy/OR exp *patient referral/OR exp consultation/AND exp delayed diagnosis/OR } \\
\text { Primary delay.mp. OR Secondary delay.mp. OR tertiary delay.mp. OR health care system/OR health care system delay.mp. OR } \\
\text { timeliness.mp. OR Patient interval.mp. OR Patient delay.mp. OR Clinician delay.mp. OR Physician delay.mp. OR delayed lung } \\
\text { cancer diagnosis.mp. OR time to diagnosis.mp. OR time to treatment.mp. or *time to treatment/OR Treatment initiation.mp. } \\
\text { OR treatment delay.mp. OR *hospital admission/OR Help seeking intervals.mp. OR Lung cancer Survival.mp. OR lung cancer } \\
\text { prognosis.mp. AND limit } 41 \text { to (human and English language and last } 20 \text { years) }\end{array}$ \\
\hline
\end{tabular}

PsycINFO exp neoplasm/OR (Lung Neoplasms or (lung adj3 neoplasm)).mp. ( $\mathrm{mp}=$ title, abstract, heading word, table of contents, key concepts, original title, tests \& measures) OR (lung cancer or (lung adj3 cancer)).mp.(mp=title, abstract, heading word, table of contents, key concepts, original title, tests \& measures) OR Respiratory tract cancer.mp. OR Bronchogenic carcinoma.mp. OR Non-Small-Cell Lung Cancer.mp. OR Non-Small-Cell Lung Carcinoma.mp. OR Small Cell lung Cancer.mp. OR Small Cell lung Carcinoma.mp. OR (Lung cancer symptom* or (lung cancer adj3 symptom*)).mp. ( $\mathrm{mp}=$ title, abstract, heading word, table of contents, key concepts, original title, tests \& measures) AND physicians/or exp family physicians/or exp general practitioners/ OR (General Practitioner* or General practice or Family Practice or Family Physician*).mp. OR (Primary healthcare or Secondary healthcare or Tertiary healthcare).mp. OR (Public hospital ${ }^{*}$ or Private hospital ${ }^{*}$ or Special hospital ${ }^{*}$ or Cancer hospital ${ }^{*}$ or Cancer Center* or cancer centre*).mp. ( $\mathrm{mp}=$ title, abstract, heading word, table of contents, key concepts, original title, tests \& measures) OR exp palliative care/OR Cancer Palliative care.mp. OR (Pulmonologist* or oncologist* or thoracic surger*).mp. $\left(\mathrm{mp}=\mathrm{title}^{\star}\right.$, abstract, heading word, table of contents, key concepts, original title, tests \& measures) OR (Thoracotom* or Lung lobectom* or Pneumonectom*).mp. ( $\mathrm{mp}=$ title, abstract, heading word, table of contents, key concepts, original title, tests \& measures) OR (Cancer surgical resection* or Surgical resection*).mp. OR (Referral or consultation).mp. OR ((Healthcare adj2 delivery) or patient admission).mp. (mp=title, abstract, heading word, table of contents, key concepts, original title, tests \& measures) AND (Diagnostic timeline* or Timeliness).mp. OR (((early detection adj3 cancer) or delay* detection) adj5 cancer).mp. ( $\mathrm{mp}=$ title, abstract, heading word, table of contents, key concepts, original title, tests \& measures) OR (Primary delay* or Secondary delay ${ }^{\star}$ or Tertiary delay* or Health system delay*).mp. OR (Patient interval* or Patient delay* or Clinician delay* or Physician delay*). $\mathrm{mp}$. OR Referral delay ${ }^{*} . \mathrm{mp}$. OR ((diagnos* adj3 delay*) or diagnostic evaluation).mp. ( $\mathrm{mp}=$ title, abstract, heading word, table of contents, key concepts, original title, tests \& measures) OR ((time adj3 treatment) or treatment initiation).mp. (mp=title, abstract, heading word, table of contents, key concepts, original title, tests \& measures) OR Treatment delay*.mp. OR (wait* time $^{\star}$ or wait $^{\star}$ time* interval or wait* list* or appointment).mp. (mp=title, abstract, heading word, table of contents, key concepts, original title, tests \& measures) OR Health service accessibility.mp. OR Help seeking intervals.mp. OR (Prognostic implication* or Lung cancer Survival*).mp. AND limit 38 to (human and English language and last 20 years)

CINAHL (MH 'Respiratory Tract Neoplasms+') OR (MH 'Lung Neoplasms+') OR (MH 'Carcinoma, Non-Small-Cell Lung/DI/DT/EP/HI/ MO/PR/RA/RT/RH/SU/SS/TH') OR (MH 'Carcinoma, Small Cell/DI/DT/EP/HI/MO/PR/RA/RT/SU/SS/TH') OR 'carcinoma, nonsmall-cell lung OR Carcinoma, Small Cell lung' OR 'lung adenocarcinoma' AND (MH 'Physicians, Family') OR (MH 'Primary Health Care') OR (MH 'Family Practice') OR 'general practitioner or gp or family doctor or primary care' OR (MH 'Secondary Health Care') OR (MH 'Multidisciplinary Care Team') OR (MH 'Tertiary Health Care') OR (MH 'Hospitals, Public') OR (MH 'Hospitals, Private') OR (MH 'Hospitals, Veterans') OR (MH 'Hospitals, Military') OR (MH 'Hospitals, Special') OR (MH 'Hospitals, Urban') OR (MH 'Hospitals, Rural') OR (MH 'Cancer Care Facilities') OR (MH 'Oncologic Care+') OR (MH 'Pulmonologists') OR (MH 'Oncologists') OR 'pulmonologist OR oncologist' OR (MH 'Surgery, Lung+') OR (MH 'Thoracic Surgery+') OR (MH 'Pneumonectomy') OR (MH 'Referral and Consultation+') OR (MH 'Patient Admission') AND 'Diagnostic timelines' OR (MH 'Early Detection of Cancer') OR 'early detection of cancer' OR (MH 'Diagnosis, Delayed') OR 'delayed diagnosis of cancer' OR 'health system delay' OR 'timeliness' OR 'timeliness in healthcare' OR 'timeliness of care' OR 'patient delay' OR 'patient interval' OR 'Physician delay' OR (MH 'Treatment Delay') OR 'diagnostic delay' OR 'diagnostic evaluation' OR 'time to treatment' OR 'treatment initiation' OR (MH 'Waiting Lists') OR 'wait` times' OR (MM 'Appointments and Schedules') OR 'prognostic implication' OR 'lung cancer survival' Limiters - English language; Published date: 19990101-20190528; human

Different databases operate in different ways. Some subject terms will be 'exploded' depending on the database to ensure capture of all relevant search topics under a given MeSH term (medical subject heading). Boolean logic and adjacencies operators will be used to combine the terms and concepts. The search terms will be modified as necessary for use in other databases; search strings developed for one database will require some modifications and might need different approaches to produce results. The research categories and subject area limiters will be used cautiously to ensure a manageable volume of literature is achieved while preserving the specificity required for this review. 
Hand searching of key journals will be done to identify any recent publications that are accepted and available online early. This will ensure articles that are not yet indexed by electronic databases are not missed. Journals containing the highest number of relevant articles will be identified by analysing the results of the database searches and will be selected for hand searching. Relevant websites will be searched to identify guidelines on standard timeframes and care pathways (eg, National Institute for Health and Care Excellence (UK), National Comprehensive Cancer Network (USA), Cancer Council Australia, European Society for Medical Oncology).

An academic health sciences librarian will be consulted on selecting the appropriate keywords and the most appropriate $\mathrm{MeSH}$ terms and filters to use in order to maximise inclusion of articles within the search and how to modify them for selected bibliographic databases. On completion, the searches from each database will be documented and references will be imported into database-specific folders in EndNote, where duplicates will be eliminated, and further screenings will be completed in Covidence.

\section{Managing references}

Search results will be imported into EndNote (V.X9), a reference management program which will store, display and enable organisation of the records specific to each database and will be used to generate the reference list for the review. Later references will be imported to Covidence, which will be used for documenting the process such as screening for eligible articles and full-text review. This is a web-based software platform developed by the Cochrane Collaboration that helps the production of reviews. While using Covidence for this scoping review, the 'Risk of Bias' portion of the application will not be used as this will be a scoping review.

\section{STAGE 3: PUBLICATION SELECTION}

Selection of publications will involve two stages. First, title and abstract will be screened against the inclusion criteria, and second, the potentially relevant papers will go through full-text review. It will be useful to examine the full paper against the inclusion criteria when a certain decision is difficult to make based on the title and abstract screening alone. To increase the reliability of the decision process, all selected papers will be independently assessed by at least two researchers. Due to the exploratory nature of this scoping review, which aims to map 'the concepts, types of evidence and gaps in research', a detailed methodological quality assessment will not be required. ${ }^{25}$

One author (AA) will perform a search of electronic database for literature. Two authors (AA and MAR) will independently review and screen the abstracts of the searched articles for inclusion. The other two authors (VL and $\mathrm{CMcD}$ ) will review the disagreements, which will be resolved by discussion with all the authors.

\section{Piloting of study selection process}

Inclusion criteria will be applied to a sample of papers to pilot the selection process in order to check that the inclusion and exclusion criteria can be interpreted consistently and that they categorise the studies correctly.

\section{Documenting decisions and disagreements}

A flow chart will be developed to display the number of publications included at each stage of the article selection process. The flow chart for reporting and presentation of systematic reviews and meta-analysis developed by the PRISMA group will be used. ${ }^{26}$ The decision to include or exclude each article will be documented in Covidence for future reference to develop the PRISMA flow chart. Studies excluded from the review will be reported with reasons for exclusion. Any disagreements will be discussed between reviewers to resolve by consensus or by arbitration by another author (VL).

\section{STAGE 4: CHARTING THE DATA}

A framework for data extraction will be used to capture the data from selected articles (online supplemental file 1). A draft of the framework has been developed, with 17 categories of content identified to be extracted. More categories will be added if identified during the final review. Relevant data will be extracted by AA. The following data will be extracted from included articles: author, publication date, study country, type/design of the study, data source, patient population, definition of timeliness used, and classification of time points or intervals in the care pathway.

\section{Piloting data charting}

The data charting will be piloted on a sample of included studies to confirm all the relevant information is extracted. The consistency of the data captured will be checked to make sure the interpretation of the columns of the data charting form and the instructions are followed properly to reduce data extraction errors. If deemed necessary, the data charting form will be revised after piloting the data charting form. Data charting will be done by one researcher independently and examined by another researcher for accuracy.

\section{Data charting process}

One author (AA) will independently perform the data extraction and results will be examined by another author or authors (VL, CL, CMcD and MAR).

\section{STAGE 5: COLLATING, SUMMARISING AND REPORTING THE RESULTS}

The selected articles will be analysed to address the three research questions. The synthesis will focus on the different definitions and terminologies used to describe timeliness in the lung cancer care pathway, and to identify timepoints and different intervals in the care seeking pathway irrespective of variation in the health systems. Results will be displayed using tables, charts or graphs as appropriate. Relevant data charted from included articles will be presented in relation to each of the review 
questions. The scoping review will summarise existing definitions, timepoints and intervals from all the selected publications and present in tabulated form. The differences and the similarities in definitions and terminologies used in Asian and Western countries will be displayed separately in tabulated form or graphs based on the typologies and interval types identified and will be discussed comparing the health system context if possible. However, the heterogenicity of the health systems and existence of medical pluralism in many developing nations in Asia may make the comparison difficult.

\section{LIMITATIONS}

From a previous literature review, it was evident that the concept of timeliness was not clearly defined in most of the timeliness of seeking care for lung cancer-related studies. It may be challenging to get a clear operational definition of timeliness from the literature included in the review. Limiting the databases included in the search, inclusion of articles only published in English, and restricting the timeframe to the last 20 years are potential limitations of this scoping review. Excluding Arksey and O'Malley's optional stage of conducting stakeholder consultation will limit this scoping review from reaching a consensus; however, the authors intend to undertake stakeholder consultation in the next phase of the research project.

\section{PATIENT AND PUBLIC INVOLVEMENT}

Patients and the public were not involved in the design or planning of the study.

\section{ETHICS AND DISSEMINATION OF REVIEW FINDINGS}

This study does not require ethical approval since the scoping review methodology aims at synthesising information from secondary data sources (publications). Dissemination of findings at relevant national and international conferences will be planned to ensure the findings from the review are brought to the appropriate stakeholders. Results will provide key information to health professionals on operational definitions of the timeliness of seeking care and to policy makers in planning, funding and delivering evidence based and effective interventions to reduce delay in seeking care and develop health systems appropriate guidelines for lung cancer care.

Contributors AA generated the idea, developed the research questions and study methods, conceptualised the review approach and developed the manuscript. AR, $\mathrm{VL}, \mathrm{CFMcD}$ and $\mathrm{CL}$ contributed to finalising the study design and research questions and supervised the manuscript preparation. All authors provided detailed comments on earlier drafts and approved this manuscript.

Funding The authors have not declared a specific grant for this research from any funding agency in the public, commercial or not-for-profit sectors.

Competing interests None declared.

Patient consent for publication Not required.

Provenance and peer review Not commissioned; externally peer reviewed.
Supplemental material This content has been supplied by the author(s). It has not been vetted by BMJ Publishing Group Limited (BMJ) and may not have been peer-reviewed. Any opinions or recommendations discussed are solely those of the author(s) and are not endorsed by BMJ. BMJ disclaims all liability and responsibility arising from any reliance placed on the content. Where the content includes any translated material, BMJ does not warrant the accuracy and reliability of the translations (including but not limited to local regulations, clinical guidelines, terminology, drug names and drug dosages), and is not responsible for any error and/or omissions arising from translation and adaptation or otherwise.

Open access This is an open access article distributed in accordance with the Creative Commons Attribution Non Commercial (CC BY-NC 4.0) license, which permits others to distribute, remix, adapt, build upon this work non-commercially, and license their derivative works on different terms, provided the original work is properly cited, appropriate credit is given, any changes made indicated, and the use is non-commercial. See: http://creativecommons.org/licenses/by-nc/4.0/.

ORCID iDs

Adnan Ansar http://orcid.org/0000-0001-6052-8657

Chaojie Liu http://orcid.org/0000-0003-0877-0424

\section{REFERENCES}

1 World Health Organization. Cancer [Internet]. Who.int, 2018. Available: http://www.who.int/news-room/fact-sheets/detail/cancer

2 International Agency for Research on Cancer, World Health Organization. World Cancer Report 2014 [Internet]. 360. Geneva, Switzerland: WHO Press, 2015. http://publications.iarc.fr/Non-SeriesPublications/World-Cancer-Reports/World-Cancer-Report-2014

3 Butler CA, Darragh KM, Currie GP, et al. Variation in lung cancer survival rates between countries: do differences in data reporting contribute? Respir Med 2006;100:1642-6.

4 Wang T, Nelson RA, Bogardus A, et al. Five-year lung cancer survival. Cancer 2010;116:1518-25.

5 Walter F, Webster A, Scott S, et al. The Andersen model of tota patient delay: a systematic review of its application in cancer diagnosis. J Health Serv Res Policy 2012;17:110-8.

6 American Society of Clinical Oncology. Thoracic cancer. Available: https://www.asco.org/research-guidelines/quality-guidelines/ guidelines/thoracic-cancer

7 National Comprehensive Cancer Network. NCCN guidelines. Available: https://www.nccn.org/professionals/physician_gls/default. aspx

8 Cancer Council Australia. Optimal care pathway for people with lung cancer. Available: https://www.cancer.org.au/content/ocp/health/ optimal-care-pathway-for-people-with-lung-cancer-june-2016.pdf

9 National Institute for Health and Care Excellence. Suspected cancer: recognition and referral. Available: https://www.nice.org.uk/guidance/ ng12/resources/suspected-cancer-recognition-and-referral-pdf1837268071621

10 European Society for Medical Oncology. ESMO clinical guideline: lung and chest tumours. Available: https://www.esmo.org/guidelines/ lung-and-chest-tumours

11 Anderson BO. Evidence-based methods to address disparities in global cancer control: the development of guidelines in Asia. Lancet Oncol 2013;14:1154-5.

12 Soo RA, Anderson BO, Cho BC, et al. First-line systemic treatment of advanced stage non-small-cell lung cancer in Asia: consensus statement from the Asian oncology Summit 2009. Lancet Oncol 2009;10:1102-10.

13 Weller D, Vedsted P, Rubin G, et al. The Aarhus statement: improving design and reporting of studies on early cancer diagnosis. $\mathrm{Br} \mathrm{J}$ Cancer 2012;106:1262-7.

14 Chandra S, Mohan A, Guleria R, et al. Delays during the diagnostic evaluation and treatment of lung cancer. Asian Pac J Cancer Prev 2009;10:453-6.

15 Dwivedi AK, Dwivedi SN, Deo S, et al. An epidemiological study on delay in treatment initiation of cancer patients. Health 2012;4:66-79.

16 Vidaver RM, Shershneva MB, Hetzel SJ, et al. Typical time to treatment of patients with lung cancer in a multisite, US-Based study. J Oncol Pract 2016;12:e643-53.

17 Arksey H, O'Malley L. Scoping studies: towards a methodological framework. Int J Soc Res Methodol 2005;8:19-32.

18 Levac D, Colquhoun H, O'Brien KK. Scoping studies: advancing the methodology. Implement Sci 2010;5:69.

19 Peters MDJ, Godfrey C, Mclnerney P, et al. Chapter 11: Scoping reviews. In: Aromataris E, Munn Z, eds. Joanna Briggs Institute 
Reviewer's Manual. The Joanna Briggs Institute, 2017. https:// reviewersmanual.joannabriggs.org/

20 Centre for Reviews and Dissemination. Systematic Reviews CRD's guidance for undertaking reviews in health care [Internet. York: Centre for Reviews and Dissemination, University of York, 2009. https://www.york.ac.uk/media/crd/Systematic_Reviews.pdf

21 Tricco AC, Lillie E, Zarin W, et al. PRISMA extension for scoping reviews (PRISMA-ScR): checklist and explanation. Ann Intern Med 2018;169:467.

22 Yankelevitz DF, Henschke Cl. Advancing and sharing the knowledge base of CT screening for lung cancer. Ann Trans/ Med 2016;4:154.
23 Yang S, Zhang Z, Wang Q. Emerging therapies for small cell lung cancer. J Hematol Oncol 2019;12:47.

24 Jones GS, Baldwin DR. Recent advances in the management of lung cancer. Clin Med 2018;18:s41-6.

25 Colquhoun $\mathrm{HL}$, Levac D, O'Brien KK, et al. Scoping reviews: time for clarity in definition, methods, and reporting. J Clin Epidemiol 2014:67:1291-4.

26 Moher D, Liberati A, Tetzlaff J, et al. Preferred reporting items for systematic reviews and meta-analyses: the PRISMA statement. BMJ 2009;339:b2535. 